Illinois State University

ISU ReD: Research and eData

Theses and Dissertations

$10-4-2015$

\title{
Using An Agent-Based Model To Study The Effect Of Reproductive Skew On Mongoose Populations
}

Stacy Lee Mowry

Illinois State University, smowry@ilstu.edu

Follow this and additional works at: https://ir.library.illinoisstate.edu/etd

Part of the Applied Mathematics Commons, and the Zoology Commons

\section{Recommended Citation}

Mowry, Stacy Lee, "Using An Agent-Based Model To Study The Effect Of Reproductive Skew On Mongoose Populations" (2015). Theses and Dissertations. 470.

https://ir.library.illinoisstate.edu/etd/470

This Thesis is brought to you for free and open access by ISU ReD: Research and eData. It has been accepted for inclusion in Theses and Dissertations by an authorized administrator of ISU ReD: Research and eData. For more information, please contact ISUReD@ilstu.edu. 


\title{
USING AN AGENT-BASED MODEL TO STUDY THE EFFECT OF REPRODUCTIVE SKEW ON MONGOOSE POPULATIONS
}

\author{
Stacy L. Mowry
}

\section{Pages}

Reproductive skew is the name given to the unequal partitioning of breeding within social groups. Within these groups, a mating hierarchy emerges wherein one dominant mating pair holds an unproportional majority of the group's reproductive benefit, while other members mate infrequently, yet allocate energy and resources toward the offspring of the dominant group members. In this paper, we use an agent-based model, which mimics mongoose populations, to investigate how reproductive skew affects the speed of natural selection, and thus how reproductive skew affects how quickly individuals within a population can become adapted to their environment. The results of the model show that due to the geometric structure of skewed breeding colonies, reproductive skew does increase the rate of natural selection. This result is consistent with the constructal law theory. Our results shed some light on skew theory and additionally have possible practical implications in conservation biology and artificial intelligence, through the genetic algorithm.

Keywords: Reproductive Skew, Constructal Law, Natural Selection, ABM, Mongooses 


\title{
USING AN AGENT-BASED MODEL TO STUDY THE EFFECT OF REPRODUCTIVE SKEW ON MONGOOSE POPULATIONS
}

\author{
STACY L. MOWRY
}

A Thesis Submitted in Partial Fulfillment of the Requirements for the Degree of MASTER OF SCIENCE Department of Mathematics ILLINOIS STATE UNIVERSITY 
(C) 2015 Stacy L. Mowry 


\title{
USING AN AGENT-BASED MODEL TO STUDY THE EFFECT OF REPRODUCTIVE SKEW ON MONGOOSE POPULATIONS
}

\author{
STACY L. MOWRY
}

COMMITTEE MEMBERS:

Olcay Akman, Chair

Lester Caudill

Dan Hrozencik

Mike Plantholt

Scott Sakaluk 


\section{CONTENTS}

\section{CONTENTS}

Page

TABLES

FIGURES

ii

CHAPTER

I. INTRODUCTION 1

II. AGENT BASED MODELS 5

Overview $\quad 5$

NetLogo 6

$\begin{array}{ll}\text { III. OUR MODEL } & 7\end{array}$

Why ABM? $\quad 7$

Turtle Breeds and Variables $\quad 9$

Set Up 11

Stochasticity 11

$\begin{array}{ll}\text { IV. ANALYSIS } & 17\end{array}$

$\begin{array}{ll}\text { Verification } & 17\end{array}$

Sensitivity 18

Methods 19

Results 20

$\begin{array}{ll}\text { V. DISCUSSION } & 23\end{array}$

$\begin{array}{ll}\text { Conclusions } & 23\end{array}$

Future Work 24

Limitations $\quad 24$

$\begin{array}{ll}\text { REFERENCES } & 25\end{array}$

$\begin{array}{ll}\text { APPENDIX A: Netlogo } & 27\end{array}$

APPENDIX B: Glossary 33 


\section{TABLES}

Table

1. Commands

2. Summary Statistics

3. Test Results
Page

12

20

22 


\section{FIGURES}

$\begin{array}{lll}\text { Figure } & \text { Page }\end{array}$

1. Initial Netlogo Setup 11

2. Extremes 18

3. Sensitivity Results 18

$\begin{array}{ll}\text { 4. BTV vs. Time } & 19\end{array}$

5. Variance of Runs with and without Skew 20

6. Frequency Distributions of BTV with and without Skew 22

7. Confidence Intervals of Mean BTV 22 


\section{CHAPTER I}

\section{INTRODUCTION}

There are many animals that perform instinctual behavioral patterns or rituals, specifically, social animals. Social mammals are those that interact highly with conspecifics, some of the most well known include bats, monkeys, elephants, dolphins, whales, meerkats, lions and mongooses. When examining the intricate social structures of these creatures a few natural questions arise: Do social mammals interact with group members in a predictable or deterministic manner? Can we use various characteristics of individuals to predict that individual's behavior? Is there any underlying order to behavior or are behavioral patterns simply coincidental? As in any scientific investigation, the first step in the investigative process is to identify a particular pattern. In this paper, we specifically aim to study the behavioral pattern of cooperative breeding, also known as reproductive skew, an almost universal characteristic of animal groups seen among fish, birds, primates and mammals.

Reproductive skew is the name given to the unequal partitioning of breeding within social groups. Within these social groups, repeatable mating hierarchies emerge wherein one dominant mating pair holds an unproportional majority of the group's reproductive benefit, while other members mate very infrequently, or not at all, yet allocate energy and resources towards the offspring of the dominant group members. The major evolutionary questions are why does this structure form, and how is it maintained, despite the obvious disadvantage to non-dominant group members. Currently, skew theory states that each group member receives a fitness advantage from living within its 
social mating group higher than the projected fitness of living, and mating, outside of the group, causing social mating to be advantageous for all group members. While a plethora of current skew models, from the transactional type to the compromise type, (Nonacs) exist within the literature, much uncertainty still prevails throughout the field. The majority of the confusion regarding skew originates from three sources. First, different models make different assumptions. Thus far, skew theory has mainly used a game theoretic approach to generate predictions about reproductive shares. Each individual has a strategy, and within this strategy each model must make certain assumptions. For example, compromise models assume a linear pay-off between competition and reproductive share, and do not hold if the pay-off is not directly linear. The difference in assumptions causes different models to generate conflicting predictions regarding skew. Next, the models may be difficult to utilize: it may be very difficult or impossible to determine the true relationship between effort devoted to competition and reproductive share gained, and there is little evidence that individual group members routinely evaluate patterns of relatedness or competitive ability in order to set reproductive skew. Finally, the models have generally poor explanatory power. For example, the models fail to answer why subordinates stay in groups that do not maximize their fitness and cannot explain differences in skew across groups in the same population.

Overall, mating hierarchies are prominent, but not fully understood. Most generally, through this project, we hope to gain an understanding of the consequence of mating hierarchies on the speed of evolution. Our investigation was inspired by the fractal structure of the mating hierarchies and the constructal law, proposed by Adrian Bejan in 1996 (Bejan).

According to Bejan, the constructal law is a universal and unifying theory of generative design in nature. The theory states that all living systems must evolve in a way that promotes optimization, by allowing a current to more easily pass through them, where a current is defined in its normal sense as a flow through a system. Examples of currents include water in a river or a 
tree, cars on a highway, or genes in a population. The constructal law predicts that when social organisms are the medium for which the current must pass, hierarchies should emerge naturally because a hierarchical structure most effectively facilitates flow of the current through the system. Examples of this phenomenon exist both within nature and human life, in any system that flows, evolves, or spreads, such as river basins, blood vascularization, atmospheric and ocean currents, animal life and migration. (Bejan). To further clarify, consider the structure of a river basin: in the middle of the system is one large, main, drainage funnel, branched off of the central drainage funnel runs a series of smaller sub-basins, following a hierarchal pattern. The hierarchical structure of the river basin is the most effective way for high quantities of water to be reabsorbed into the earth. Or, in other words, the hierarchical structure must evolve, because this structure promotes optimization. Just as river basins are natural systems that water flows through, populations are natural systems that genes flow through. If Bejan's hypothesis, that the constructal law directs design in nature, is correct, then the hierarchical structure of skewed breeding should increase the efficiency of gene transfer, and hence speed up the process of natural selection within a population. If natural selection is able to work more quickly on a population we would expect individuals within the population to become more adapted to their environment more quickly over generational time.

In this model, we will examine specifically two species of mongooses found on the Mweya Peninsula in Queen Elizabeth National Park in Uganda, although we could have chosen a wide variety of other social mammals. These closely related species, which otherwise are strikingly similar in their ecology and behavior, differ markedly with respect to skew. The dwarf mongooses typically have a single breeding female per group, while almost all reproductively mature banded mongooses can breed. According to a previous study Behavioural and endocrine mechanisms of reproductive suppression in Serengeti (Creel) the alpha females, those which control breeding and initiate pack movement, within a 
population of dwarf mongooses accounted for 219 of the 304 pregnancies (about $80 \%$ ) despite subordinates outnumbering alpha females by more than two to one. Overall, only about $25 \%$ of all females, within a pack of dwarf mongooses, breed. The banded mongooses, on the other hand, have an egalitarian breeding society in which most females breed in each attempt. According to the same study, over $80 \%$ of females conceived during a breeding period with about $25 \%$ of the males in the pack. This example begs for an explanation. What exactly causes the vast differences in skew?

Overall, we aim to use the models to exam how these different reproductive strategies affect the speed of natural selection, which affects how quickly a beneficial trait can spread to fixation within a population. We expect, that due to the hierarchical structure of skewed breeding groups, cooperative breeding will increase the rate of natural selection. We make no claims that reproductive skew increases and individual's fitness, this is a much more intricate biological question that provokes issues of how and why natural selection works on the individual level. Instead, we aim to use the model to show that there is a correlation between cooperative breeding and the rate of natural selection, due to the geometric structure of the mating hierarchy, and consider possible implications of this result. 
CHAPTER II

\title{
AGENT BASED MODELS
}

\begin{abstract}
Overview
Agent-based modeling is a method of modeling complex systems based on the interactions of autonomous agents in a particular environment, especially useful in biology, ecology and the social sciences. Agents within an agent-based model (ABM) can be heterogeneous. This means each agent has a unique set of characteristics (such as: unique variability in space, life cycle details, phenotypic variation, experience, learning, genetics, evolution, etc.) that determine that agent's particular behaviors and interactions with other agents and the agent's environment. This heterogeneity of agents across a population allows for a great range of diversity within the simulation. ABMs are considered a ground-up approach. There is no central authority that controls agent behavior in order to optimize system performance and population behaviors are not explicitly programmed into the model. Instead, interactions between agents, which are encoded in algorithmic form in a computer program, and their local environments (agents may only interact with a subset of agents surrounding them in a 2D topology) gives rise to the higher order patterns, self-organization, and the behavior of the system as a whole.

ABMs are an intuitive method to study ecological systems as they allow researchers to include an array of details into their simulation, closely mimicking the population being studied. They provide a natural computer-based extension to biological experiments that are otherwise impossible to conduct in a lab or field setting because of cost, time biological or ethical constraints.
\end{abstract}




\section{NetLogo}

NetLogo is an agent-based programming language and integrated modeling environment developed by Uri Wilensky of Northwestern University. Agents within this programming environment are called turtles and the agents environment is broken into patches. Both patches and turtles can have unique characteristics, programmed by the user, and turtles can interact with other turtles as well as with patches. NetLogo is very userfriendly. The main interfaces contains a $2 \mathrm{D}$ spatial view of the model environment as well as sliders, switches, and choosers that allow the user to isolate variables and adjust model parameters, and plots and monitors that allow users to measure outputs generated from the model. NetLogo is based on an object-oriented programming language and can be downloaded for free from https://ccl.northwestern.edu/netlogo/index.shtml (Appendix 1). 


\section{CHAPTER III}

\section{OUR MODEL}

\section{Why ABM?}

The big question, which inspired this model, is how the geometric structure of cooperatively breeding animal societies affects the speed of natural selection, which, ultimately, will allow populations to more quickly adapt to their changing environments.

There are many complications that arise when attempting to empirically study natural selection. First, and most obviously, there is the issue of time. Evolution (and thus evolution by natural selection) is a slow process; it is impossible to gather observations on any evolutionarily relevant time scale. Next, there is the issue of subjectivity: how can researchers objectively measure and quantify evolutionary change via simple observation? Although gene sequencing is possible it is extremely costly and virtually impossible over such a daunting time scale. Additionally, logistically it would be equally as impossible to study a stationary population over such a long time due to immigration and emigration causing difficulties in differentiating genetic change due to natural selection from genetic change due to migration and interbreeding. For these reasons, ABMs offer a much-needed method to study evolution by natural selection.

Our goal was to study how reproductive skew, a specific attribute of a population, affects the speed of natural selection within the population, a population-level phenomenon. Furthermore, an ABM gives us the power to control for differences in the speed of natural selection due to other variations in behaviors and specifically isolate the effect of skew on the speed of natural 
selection.

An ABM suits our question over a compartmental model because it does not require the assumption of homogeneous populations. That is, each agent in our model could behave differently from each other agent. When studying reproductive skew, heterogeneity is a necessary condition. First, we must distinguish between behavior of dominant and subordinate group members. Next, we must be able to account for conflicts between individual members of each group, so we must be able to account for within-group heterogeneity within the model. For example, a healthier group member is more likely to fight for dominance than a less healthy group member. An ABM allows us to account for the differing behaviors based on different characteristics.

Our model mimics two species: the banded mongoose, and the dwarf mongoose. All of the behaviors in the model, which we will further describe in the paper, are modeled after an extensive literature review. We would quickly like to address the output variable, "Beneficial Trait Value" (BTV), within the model. BTV in this model is solely the name of a turtle characteristic that is used to quantitatively measure the speed of natural selection. If we were to give it some biological relevance we may think of it as how suited an individual is to his or her environment. For example, it could represent any quality that would grant the individual greater survival probability and consequently greater reproductive success such as: running speed, ability to camouflage, foraging ability, etc. 
Turtle Breeds and Variables

There are 7 globalvariables in the model:

- aggression

- capacity

- challenge-probability

- elders

- females-challenge

- males-challenge

- skew

Aggression determines the rate of aggression between groups when their territories overlap. High aggression means younger turtles are more likely to die when the groups confront one another. Aggression is a slider from 0 to 100 on the interface. Capacity determines the carrying capacity, or the maximum number of individuals that can be sustained, of the population. It is a slider on the interface from 0 to 360 . Challenge probability can be determined by the user and is represented by a slider between $1 \%$ and $10 \%$, which determines how likely non-dominant members are to win a challenge against a dominant member. Elders is a slider between 0 and 1 , which determines the weight within the rank function specifying the power elders hold within thehierarchy:

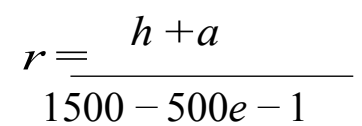

Where $\mathrm{r}=$ rank, $\mathrm{h}=$ health $\mathrm{a}=$ age and $3=$ elders. For example, if elders is set at 0 , elders do not have that much power. This means that more healthy young females within a group are more likely to inherit dominance than older, less healthy females. On the other hand, if elders is 1, older less healthy females are more likely to inherit the dominance than younger, more healthy females. Females-challenge and males-challenge determine the probability that a nondominant female or a non-dominant male, respectively, will challenge a dominant male or female for his or her breeding dominance. We must note, females-challenge is only relevant when skew is on. 
Finally, skew determines whether or not the population exhibits reproductive skew among the females. When skew is on, the dominant females gets the majority of the reproduction, while the majority of the other females do not breed at all. When skew is off the females breed egalitarianly. Skew is an on off switch on the user-interface. We chose to parameterize these values since they are not explicitly known in order to study the effect of each within a biologically reasonable range.

There are two breeds of turtles: male and female, the males are represented as side-ways facing mice and the females are represented as forward facing mice. Each turtle has 12 turtle variables:

- age

- challenging

- dominant

- BTV

- group

- group-size

- health

- mutated

- oestrus

- oldest

- rank

- time-in-hab

Each will be described later in the code description. 


\section{Set Up}

The turtles begin in 6 groups of 32. This is intended to mimic the population density on the Mweyan peninsula. The groups are initially not evenly spaced out, to represent a more realistic population. Each group is a different color so they may be easily differentiated and ungrouped turtles will turn yellow. Each group has one dominant male and one dominant female. The initial turtles have varying ages between 0 and 3600 but the age structure is consistent across the groups. Each turtles begins with a random BTV value between 0 and 4 , determined by the built-in command $\operatorname{random}(n)$ with $n=5$.

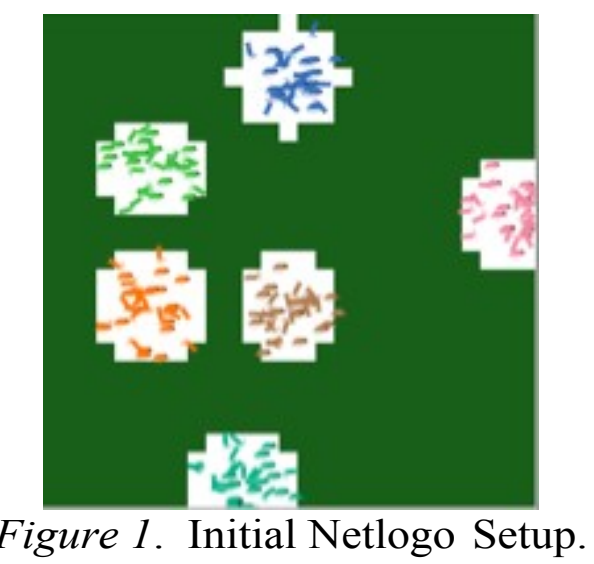

\section{Stochasticity}

All stochasticity in the ABM was included using the built-in $\operatorname{random}(n)$ command and the one - of command. According the the random $(n)$ command, the program then will choose a random integer $i$ such that $0<i<n$, where each $i$ has an equal probability of being selected. The built-in one-of command randomly chooses one turtle, or one patch, each with an equal probability. 
Table 1

Commands

Command

Relocate/Relocate2

Inherit

Challenge

Emigrate

\section{Description}

Turtles move habitat every 5

days. The white patches around the groups represent each group's habitat.

When the breeding female reaches an age of 3333, she loses her dominance and a female in the group with the highest rank inherits it.

If a non-dominant male or female has a higher health than the dominant male or female he or she will challenge the dominant member of his or her group with a certain probability. When a male or female challenges the dominant member of his or her group, he or she has a certain probability of winning and gaining reproductive dominance. Otherwise, the challenging individual loses health Only females in a skewed population challenge.

If there are more than 7 males in the group, young males in that group will emigrate when they are 850 days old.

(Table Continues) 
Exile

Disperse

Switch

OldAge

Poor Health

Join

Move Turtles
If a group becomes too large ( $>$ 58 members) the dominant female will exile a random male and female.

If a group becomes too small $(<4$ reproductive males or females) the entire group will break up and each member will search for a new group.

When the dominant male dies, a random male in the group will become the new, dominant male.

Turtles die on tick 3650 .

A turtle will die if his or her health reaches zero.

When an ungrouped turtle turtle comes in contact with a group he or she will always join the group.

Move is used to initially assemble the turtles. 
Command

Carrying Capacity

Form

Set Oestrus
Description

Since the model is theoretical it seemed unnecessary and computationally wasteful to program in realistic elements such as food source, and other resources. Instead, carrying capacity models the limited resources of the population

A carrying capacity considers the natural effect that there is only a finite amount of food and resources available and therefore the environment can only support a finite population. Adding a carrying capacity function limits the population from growing exponentially. According to this function if the population exceeds the carrying capacity, determined by the user, then a random ungrouped turtle and a random grouped turtle with low health will die.

If there are at least three ungrouped, reproductively mature females, and at least four ungrouped, reproductively mature males, they will form a new group.

Females can reproduce once they are a year old, in which case oestrus is set from 0 to 1 .

(Table Continues) 
Set $\mathrm{Hab}$

Set Mutated

Update Dom

Set Rank

Update Size

Reproduce-a (skew)
The white group of

patches around a group

represent its habitat.

If an offspring has a genetic mutation his or her BTV will be updated. $90 \%$ of mutations are deleterious.

Update-dom accompanies the challenge command. When a lower ranked member challenges the dominant male or female in the group and the lower ranked member wins the challenge.

Set rank assigns a rank to every female.

Update size keeps track of each turtle's group size.

Each group has a new litter on the 1st, 62nd and 123rd day of each year. When skew is on the oldest female in each gr group has $75 \%$ of the offspring. There is a $75 \%$ chance the dominant male will be the father and a $25 \%$ chance any other male will be the father. The offspring inherits the average BTV of his or her parents. 
Reproduce-b (with skew on)

Reproduce-a (with skew off)

Reproduce-b (with skew off)
When skew is on a random female has $25 \%$ of the groups littler. There is a $75 \%$ chance that the dominant male will be the father and a $25 \%$ chance that a random male will be the father.

When skew is off the dominant male has $75 \%$ of the matings with any female.

When skew is off $25 \%$ of the matings go to a random male and a random female. 


\section{CHAPTER IV}

\section{ANALYSIS}

\section{Verification}

Since this model is answering a theoretical question and not intended to generate any exact quantitative output we focus on model verification over model validation. Model verification is a process that determines whether the programming implementation of the abstract conceptual model is correct. (Bharathy) To do this we run 30 trials in each of the two extreme cases. Extreme cases are model runs with all of the parameters set on their absolute minimum or maximum values. The first case we set all parameters to values expected to expedite natural selection. For example, BTV determines inheritance of dominance over age, non-dominant males and females challenging a less healthy dominant member in their group had the maximum likelihood (10\%) of winning the challenge. There was no aggression or conflict between groups and the population did exhibit skew. On the other extreme end, we set all parameters to values likely to deter the speed of natural selection. In this scenario, age determined inheritance of dominance over health. Challenging males only had the minimum likelihood (1\%) chance of winning a challenge against less healthy dominant members for mating privilege, groups were very aggressive, and the population did not exhibit skew. As expected these conditions gives us very high BTV values in the first scenario $($ mean $=10.53)$ and very low BTV values in the second scenario $($ mean $=4.68)$. Means and 95\% CIs are shown in Figure 2. 


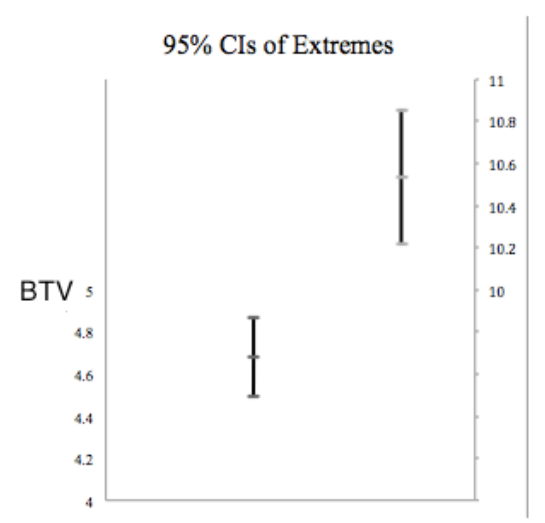

Figure 2. Extremes.

\section{Sensitivity}

We analyzed the sensitivity of the parameters we believed to be most influential in the model using a one-way, variance-based analysis. In order to determine a parameters sensitivity we kept all other parameters constant at their middle values with skew on and varied each parameter of interest, collecting data for very low, low, high and very high values. Our results, which are summarized in Figure 3, determine that challenge-prob had the largest effect on BTV. The range of challenge-prob values yielded the largest variance in BTV outcome. The BTV outcome was less sensitive to changes of the elder and aggression parameters. A similar method could be used to determine the sensitivity of all parameters not included within the scope of our analysis.

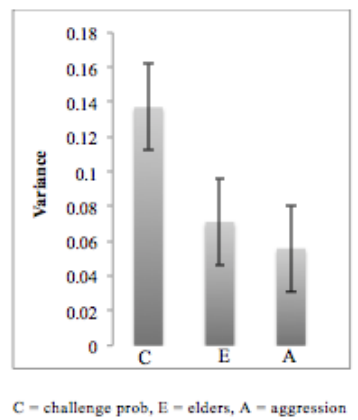

Figure 3. Sensitivity Results. 


\section{Methods}

We aim to compare the mean BTV of populations exhibiting skew and the mean BTV of populations not exhibiting skew to determine the effect of skew on BTV. To collect our data we run each simulation 150 times for 500 years. In order to determine the length in simulated time of each of our runs we first run each model (skew and no skew) for 1000 years. This test illustrated that BTV increased at a constant rate when skew was both on and off (Figure4).

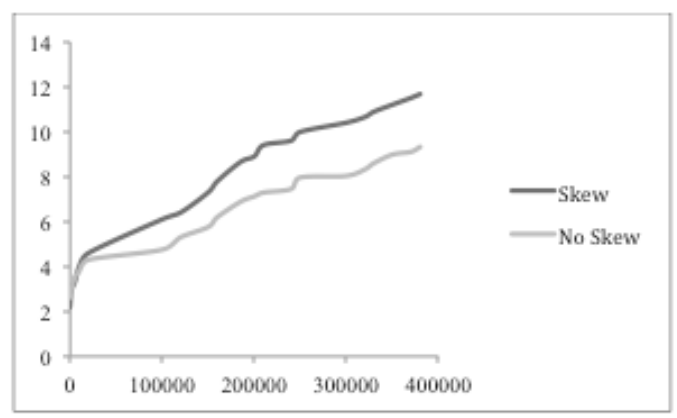

Figure 4. BTV vs. Time.

We therefore decided to run each simulation for 500 years in order to save computational resources, while still studying an evolutionarily relevant time scale. Next, we had to determine how many data points to collect for our study. To do this we analyzed the change in variance between subsequent trials by adding an additional data point. For example, we ran our initial two simulations and calculated the variance of the two data points. We then ran a third simulation and calculated the variance of the three data points. We continued this process until the variance became constant. Or, in other words, we continued until we were not gaining any additional information from collecting more data. We, however, did have the requirement that we must collect the same amount of data on populations with skew and populations without skew. Therefore, we had to continue collecting data until the variance between 
runs of both skew and no skew became relatively constant. This occurred after 150 runs of each (Figure 5). All data was collected using the built-in Netlogo application Behavioral Space (see Appendix). All statistical analysis was done with the Excel package Real Statistics.

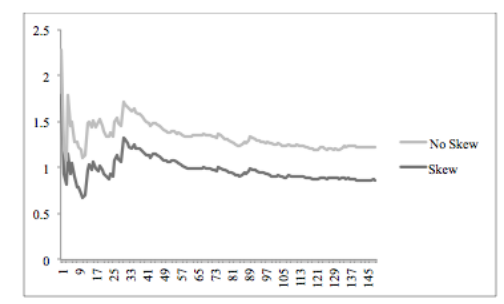

Figure 5. Variance of Runs with and without Skew.

Results

The results from our trials concluded that the mean BTV of populations with skew after 500 years was significantly higher than the mean BTV of populations without skew after 500 years. The summary statistics are given in Table 2, frequency distributions are displayed in Figure 6, and 95\% CI's of mean fitness with and without skew are displayed in Figure 7.

Table 2

Summary Statistics

$\begin{array}{lll} & \text { Skew } & \text { No Skew } \\ \text { Range } & 6.48-11.72 & 5.81-8.82 \\ \text { Median BTV } & 8.47 & 7.03 \\ \text { Mean BTV } & 8.54 & 7.05 \\ \text { Standard Deviation } & .929 & .595 \\ \text { Sample Size } & 150 & 150 \\ \text { Standard Error } & .076 & .049\end{array}$

To analyze the statistical significance of our results we performed a Welch's t-test to compare the means of our two samples. Under Welch's t-test we assume that both samples come from a normal probability distribution, however, 
unlike a regular pooled t-test we do not need to guarantee that the variance of the two samples are equal. We checked the normality assumption using a Shapiro-Wilkes test, which generated p-values of .98 and .976, therefore we can safely assume that both samples come from a normally distributed population. As listed in the summary statistics the variance of our first data set is .863 , while the variance of our second data set is .354 (Table 1 ). We performed an F-test to determine whether these variances of each data set are statistically different. This test resulted in a p-value of $8.97 * 10^{-} 8$, we therefore have sufficient reason to reject the null hypothesis that the variances between samples are the same for the alternative hypothesis that the variances between the samples are statistically different. Due to this difference in variance between the samples, we perform the Welch's t-test. Our Welch's ttest gave us a p-value less than 0.0001 . By conventional criteria, this difference is considered to be extremely statistically significant. Therefore, we can reject the null hypothesis that the sample means are equal. It is evident that BTV among trials with skew is significantly higher than fitness of groups without skew. This result is further emphasized by the non-overlapping $95 \%$ CI's given in Figure 7. We therefore can conclude that natural selection is able to act more quickly on groups exhibiting reproductive skew and thus skewed breeding has a positive impact on BTV. 

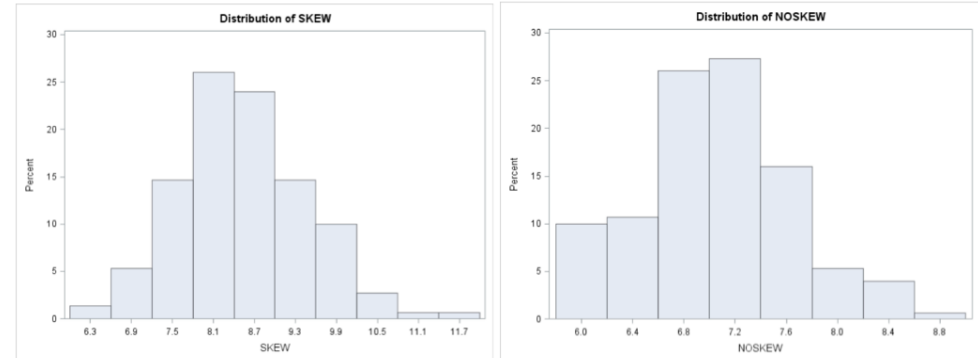

Figure 6. Frequency Distributions of BTV with and without Skew.

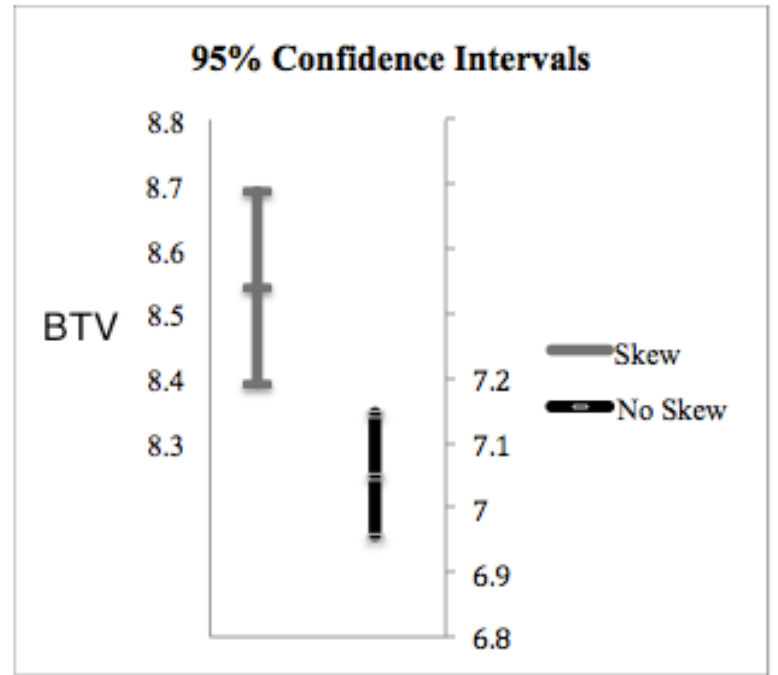

Figure 7. Confidence Intervals of Mean BTV.

Table 3

Test Results

\begin{tabular}{|c|c|}
\hline Test & $\underline{\text { Result }}$ \\
\hline F-test & $<.0001$ \\
\hline Shapiro Wilkes & .978 (no skew) .985(skew) \\
\hline
\end{tabular}




\section{CHAPTER V}

\section{DISCUSSION}

\section{Conclusions}

The results of our model concluded that a skewed breeding system does affect the speed of natural selection in that groups that have a skewed mating structure will more quickly be able to evolve beneficial traits than groups that do not have a skewed mating structure. This means that skewed populations should be more able to adapt to a changing environment. This result holds importance from a conservation perspective in lieu of climate change and habitat shift. Perhaps species that have a skewed breeding system will be more likely to survive given current issues of global warming, and able to adapt to their rapidly varying habitat. This information could be useful to conservation agencies in deciding how to optimally allocate funds.

Furthermore, our results may hold implications in light of the constructal law. As stated, Bejan proposed that all natural systems should evolve to have a certain structure that facilitates flow. Skewed breeding societies do in fact have the tree-like structure proposed by Bejan, where hierarchies emerge naturally, and as we have shown, this geometric structure does facilitate gene flow. If Bejan's theory is universal, and our results support his theory, then it seems that reproductive skew evolved because is simply following the natural pattern generation of all systems. Additionally, our results can have implications in computer algorithms. The genetic algorithm is a search algorithm used to optimize systems that is based on natural selection. If reproductive skew speeds up the rate of natural selection, it can possibly also be implemented in the genetic algorithm in order to make it more efficient. 


\section{Future Work}

There is room for future work within the realm of ABMs to further investigate our hypothesis. For example, our results showed that reproductive skew did indeed affect the rate of natural selection, however, we did not study how the strictness of the hierarchy affected the speed of natural selection. For example, our dominant members receive $75 \%$ of the mating, while all subordinate members split the other $25 \%$, what would happen if we made the hierarchy a stronger or weaker split? (95\% vs. 5\% or $60 \%$ vs. $40 \%$ ). Additionally, how do other environmental conditions affect our results? We discuss implications in conservation and habitat shift, can this be directly implemented into the model? Finally, how do different group sizes affect our results?

\section{Limitations}

As in every study, our study certainly had a few limitations we would like to address. First, there were of course behaviors, and environmental factors left out of our model for simplification. For example, there were no predators in our model, only a carrying capacity command. Furthermore, intergroup aggressiveness towards newborns was not modeled. (It is known that dominant females can act aggressively towards the offspring of non-dominant females in socially reproductive mammals.) Our non-grouped turtles cannot reproduce, which is unrealistic, and turtles cannot choose to leave a group. Additionally, modeling group size became unexpectedly difficult. While in natural populations groups tend to average around 20 members, all of our groups remained at their maximum size throughout the span of each run. Perhaps various probabilistic commands should have been added so group size followed a normal distribution around the average of 20 members per group. Finally, our code was not as efficient as possible. Cleaning up the code would benefit future researchers to save computational time and energy. 


\section{REFERENCES}

Bejan, A.I., Zane, P. (2012). Design in Nature: How the Constructal Law Governs Evolution in Biology, Physics, Technology and Social Organization. New York: Doubleday.

Bejan, A. (2015). Constructal Law: Optimization as Design Evolution. J. Heat Transfer Journal of Heat Transfer.

Cant, M.A. (2015). Banded Mongoose. Banded Mongoose.

Cant, M., Otali E., and Mwanguhya, F. (2002). Fighting and Mating Between Groups in a Cooperatively Breeding Mammal, the Banded Mongoose. Ethology 108.6, 541-550.

Cant, M. (2009). Reproductive Skew Theory. Reproductive Skew in Vertebrates: Proximate and Ultimate Causes By Reinmar Hager and Clara B Jones, 1-4.

Cant, M.A. (1992). Social Control of Reproduction in Banded Mongoose. Animal Behavior 59.1, 147-158.

Creel, S. , Creel, N., and Wildt, D., and Monfort, S. (1992). Behavioural and Endocrine Mechanisms of Reproductive Suppression in Serenge Dwarf Mongooses. Animal Behavior 43.1, 231-145.

Gammack, D., Schaefer, E. (2013). Global Dynamics Emerging from Local Interactions: Agent-Based Modeling for the Life Sciences. Mathematical Concepts and Methods in Modern Biology: Using Modern Discrete Models. By Raina S. Robeva and Terrell L. Hodge.

Hodge, S. (2005). Helpers Benefit Offspring in Both the Short and Long-term in the Cooperatively Breeding Banded Mongoose. Proceedings of the Royal Society B: Biological Sciences 1580, 2479-2484.

Luca, D., Ginsberg, J. (2001). Dominance, Reproduction and Survival in Banded Mongooses: Towards an Egalitarian Social System. Animal Behavior, 61.1, $17-30$. 
Nonacs, P., Hager, R. (2011). The Past, Present and Future of Reproductive Skew Theory and Experiments. Biological Reviews, 86.2,271-298.

Reeve, H., Sheng-Feng, S. (2013). Unity and Disunity in the Search for a Unified Reproductive Skew Theory. Animal Behaviour, 85.6, 1137-1144. 


\section{APPENDIX A NETLOGO}

\section{Obtaining NetLogo}

To download NetLogo go to https://ccl.northwestern.edu/netlogo/download.shtml and click Download. Once redirected to the next webpage choose the appropriate operating system, and follow the instructions given within download.

\section{Adjusting Code}

Although the program developed for this paper was intended to mimic mongoose populations, it can be edited in order to simulate and study skew within other populations. First, of course, there are seven different settings on the user interface, all which can be adjusted to match the population at hand. To adjust sliders simply click on the selector and move it left or right.

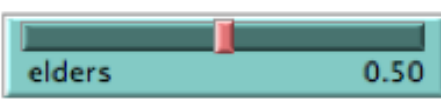

To turn skew on or off, click on the switch.

\section{】Off skew}

All settings not listed in the user interface can be adjusted directly in the code. To adjust the initial population number, edit the set-up command. 


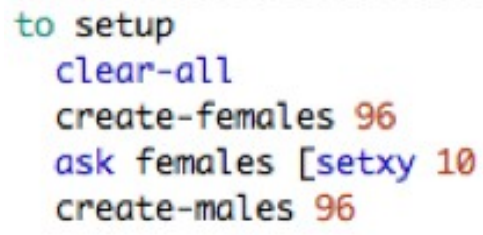

Currently the population initializes with 96 males and 96 females. To change, insert desired population size in place of 96. If your desired population is less than 96 you must not comment out unwanted turtles in the assemble command by inserting a ";" before the line of code you wish to comment out. For example, here I commented out turtles 14 and 15.

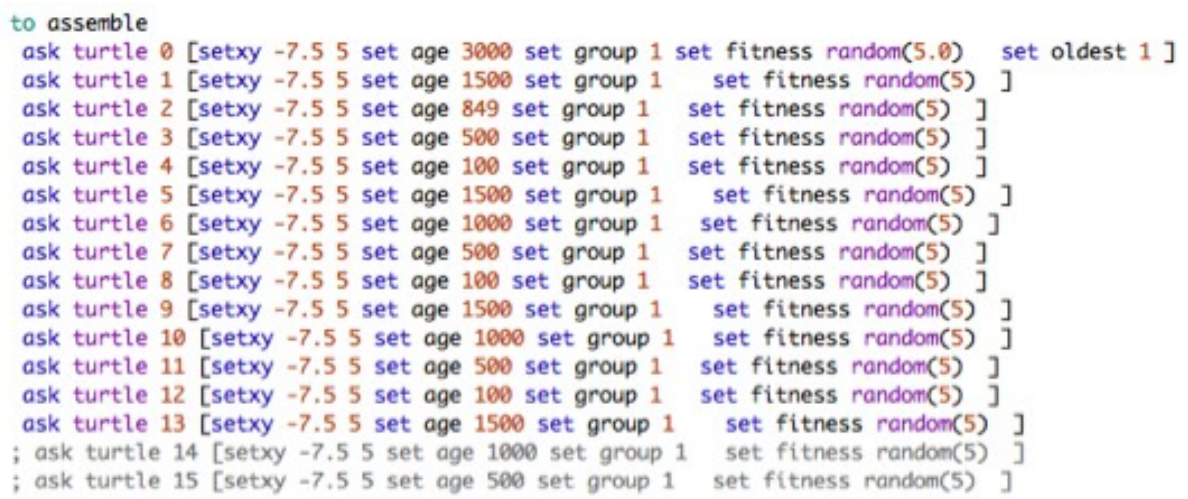

Be sure to not comment out the oldest female or dominant male within a group.

Currently, the population begins with a random distribution of turtle fitness between 0 and 4, if you would like to being with a uniform fitness, replace set fitness random(5) in assemble with set fitness $n$, where $n$ is a fitness value of your choice.

Males emigrate from their group if their group has more than 7 males, and they are 850 days old. Both these conditions can be changed by editing the emigrate command.

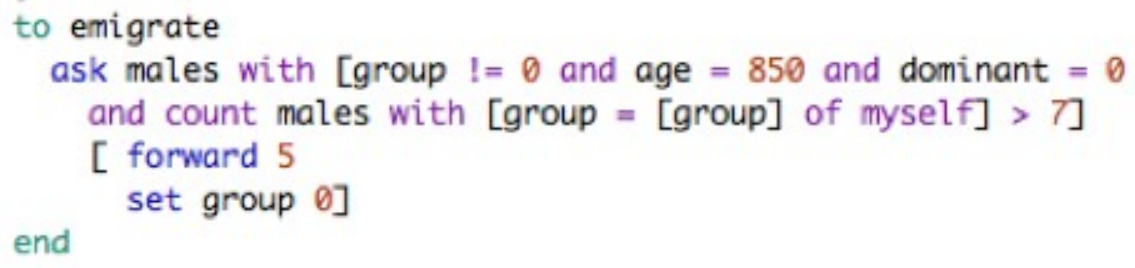


You may change the maximum group size by editing the exile command. The current maximum group size is 58 , adjust it by replacing 58 with the desired group size.

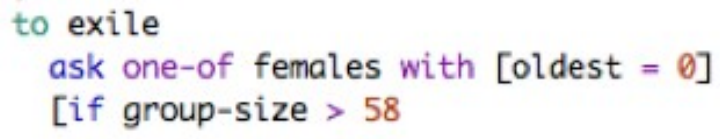

Males pass on their dominance at 3649 days, to change this replace both of the 3649's in the switch command

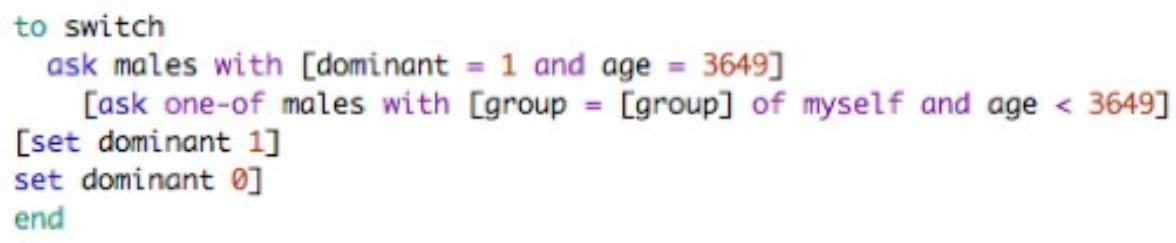

Females pass on their dominance at 3333 days, to change this replace both of the 3300's in the inherit command

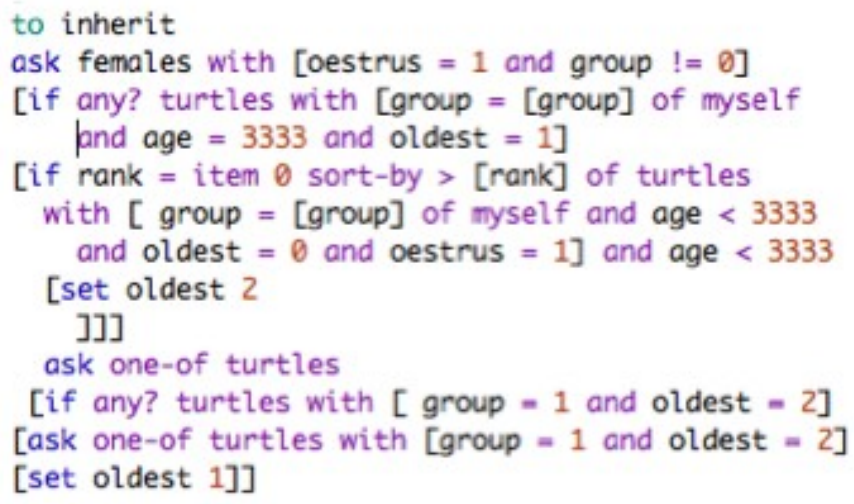

Make sure if you edit either of these conditions you ALSO edit the challenge command to match the new ages in switch and/or inherit. 


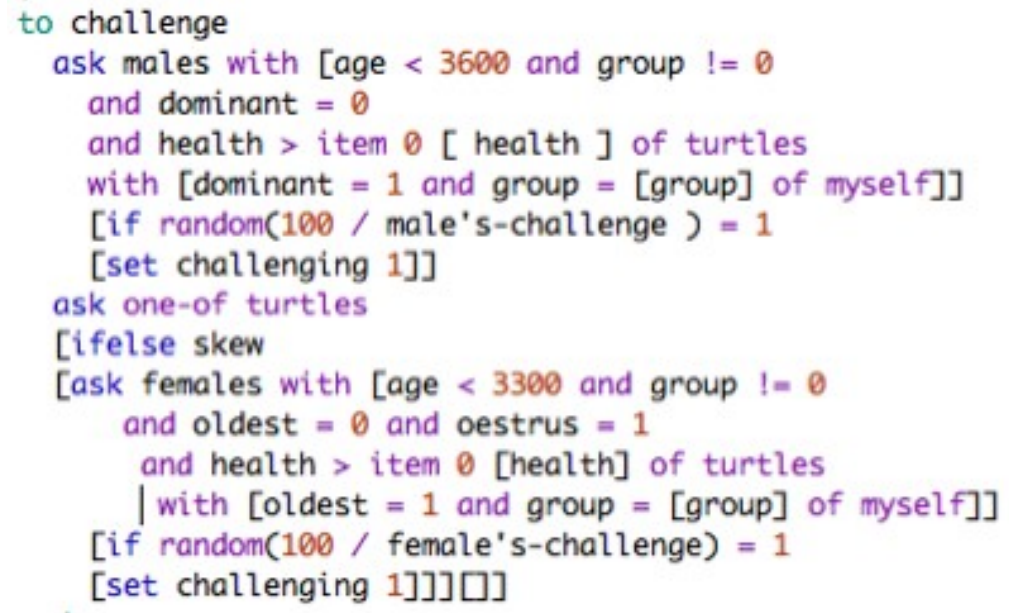

Turtles under the age of 70 days are considered infants and are likely to die due to aggression from other packs. To change the age of 70 days replace all four 70s in the challenge command with desired age.

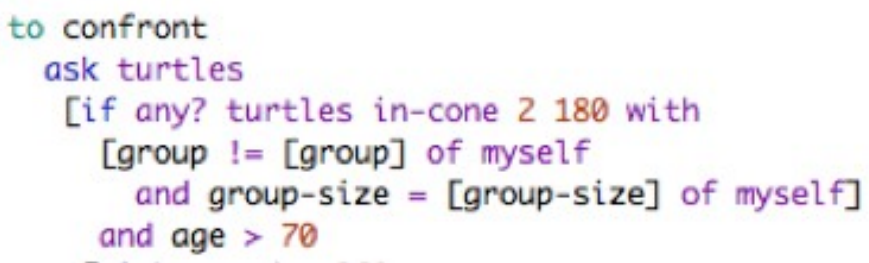

Turtles die of old age after 3650 days. This can be edited in the old-age command by replacing 3650 with desired maximum life-span

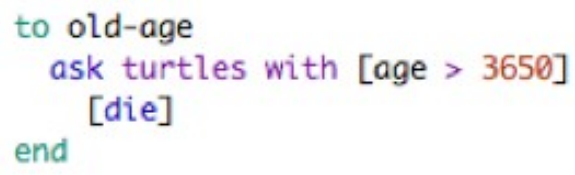

Females become sexually mature after 365 days. This can be edited in the set-oestrus command

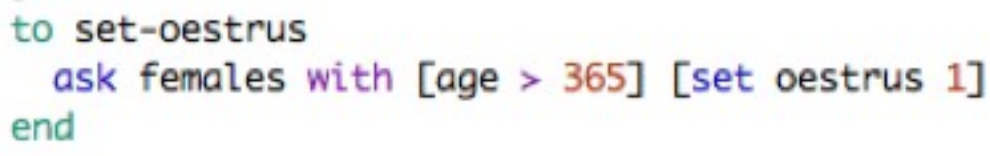


Any commands can be commented out with a ; within the to go command. Note: the program may not run properly if certain commands are commented out.

\section{Running Code}

To run the code, first adjust your sliders, then click the set up button, followed by go.

To run the code continuously right click on go, click edit, and select the forever option. The stop and start the simulation mid-run, click go. Time is measured in ticks and can be viewed directly above the world. Fitness is monitored on the Fitness plot.

\section{Collecting Data}

To have data directly sent to Excel from the simulations for analysis, use the built in NetLogo application BehaviorSpace. BehaviorSpace can be found in the Tools Menu on Netlogo. Once Behavioral Space is open, select New experiment and fill in the appropriate settings. For our paper the following BehaviorSpace conditions were used. 


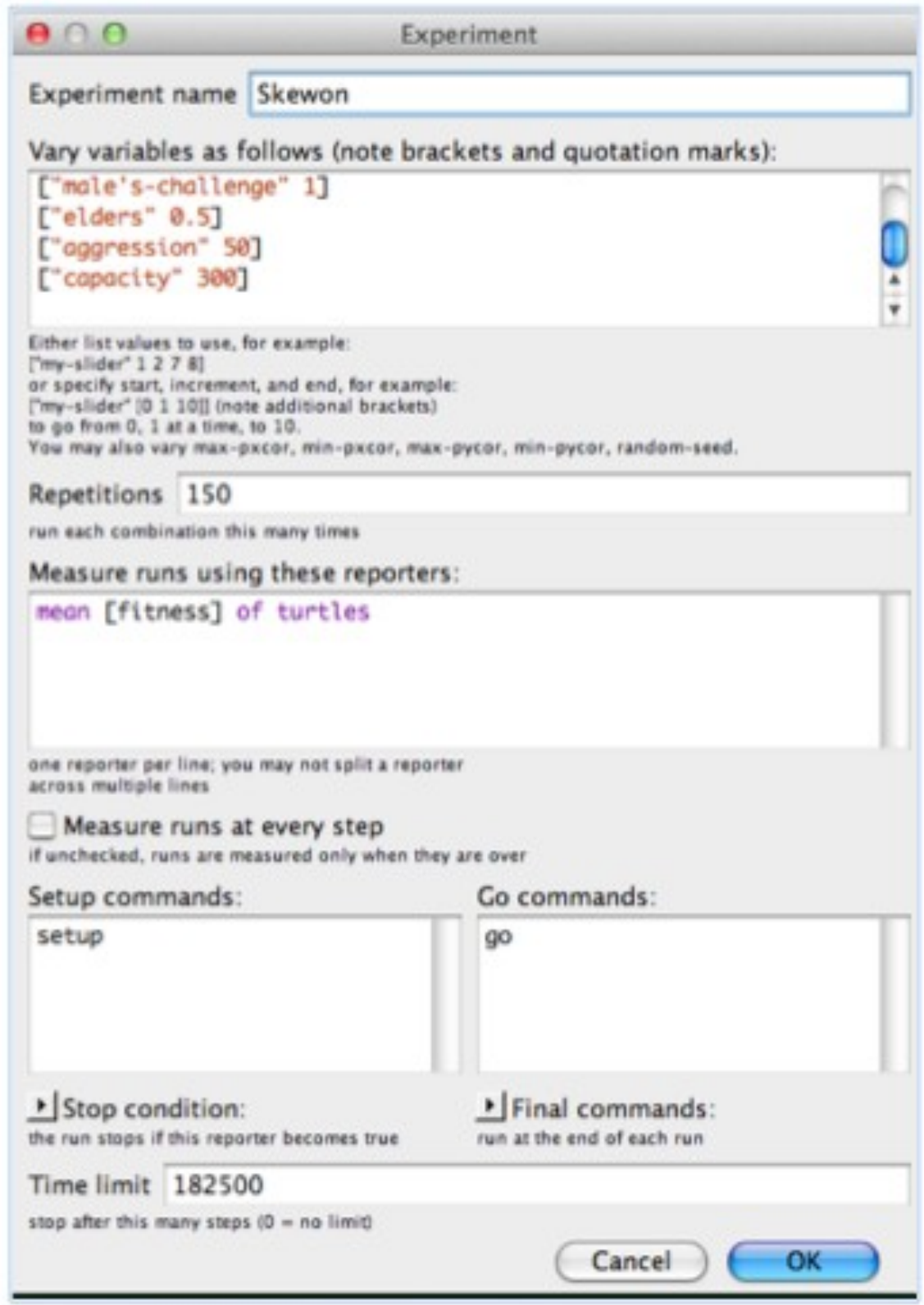




\section{APPENDIX B}

\section{GLOSSARY}

Constructal law: the constructal law was stated by Adrian Bejan in 1996 as follows: "For a finite-size system to persist in time (to live), it must evolve in such a way that it provides easier access to the imposed currents that flow through it (Bejan).

Compartmental model: A model that stratifies a population into subpopulations in order to understand complex dynamics of a system. These models utilize differential equations to model how members of the population move between subgroups.

Transactional models: Reproductive skew models that assume that reproductive share is offered as a reward from dominant group members for cooperation of subordinate group members.

Compromise models: A reproductive skew model, also known as a tug-of-war model, which assumes that reproductive shares are determined by competitive abilities of individuals.

Welch's t-test: A statistical two-sample test used to test the hypothesis that two populations have equal means. Welch's t-test is an adaptation of Student's ttest, and is more reliable when the two samples have unequal variances and unequal sample sizes. 